\title{
Qualidade física do solo sob sistemas de integração lavoura-pecuária
}

\author{
Glenio Guimarães Santos(1), Robélio Leandro Marchão(2), Euzebio Medrado da Silva(2), \\ Pedro Marques da Silveira( ${ }^{(3)}$ e Thierry Becquer(4)
}

\begin{abstract}
(1)Universidade Federal do Piauí, RodoviaBR 135,Km03,BairroPlanalto Horizonte,CEP64900-000Bom Jesus, PI.E-mail:gleniogm@ufpi.edu.br (2)EmbrapaCerrados,CaixaPostal08223,CEP73310-970Planaltina,DF.E-mail:robelio.leandro@cpac.embrapa.br,euzebio.medrado@gmail.com (3)Embrapa Arroz e Feijão, Caixa Postal 179, CEP 75375-000 Santo Antônio de Goiás, GO. E-mail: pmarques@cnpaf.embrapa.br (4)Institut de Recherche pour le Développement, UMR 210 Eco\&Sols, 2, Place Viala, F-34060 Montpellier Cedex 1, France. E-mail: thierry.becquer@ird.fr
\end{abstract}

Resumo - O objetivo deste trabalho foi avaliar a qualidade física do solo em sistemas de integração lavoura-pecuária (ILP), em comparação à pastagem contínua e ao cerrado nativo. Durante dois anos (2005 e 2006), amostras de um Latossolo Vermelho argiloso foram coletadas de duas camadas $(0-20$ e 70-80 cm), em seis áreas cultivadas em diferentes sistemas de rotação de culturas e ILP. Uma área de pastagem contínua e outra de cerrado nativo foram utilizadas como referências. Foram analisados os seguintes atributos físico-hídricos: densidade do solo, umidade de saturação, porosidade total, macroporosidade, microporosidade efetiva, saturação efetiva, condutividade hidráulica saturada de campo e de laboratório, e curva de retenção de água do solo. Todos os sistemas de cultivo provocaram impacto nos atributos físico-hídricos, na camada $0-20 \mathrm{~cm}$. O cultivo contínuo de pastagem proporcionou a melhor qualidade física do solo. Na comparação entre os anos, apenas o sistema preparo convencional do solo mostrou incremento na densidade e redução na porosidade do solo. A pastagem em rotação no sistema ILP, mesmo após quatro anos, não favorece a qualidade física do solo em comparação à pastagem contínua.

Termos para indexação: condutividade hidráulica, curva de retenção, porosidade, rotação de culturas, sistema plantio direto.

\section{Soil physical quality in integrated crop-livestock systems}

\begin{abstract}
The objective of this work was to evaluate the physical quality of soil in integrated crop-livestock systems (ICL) in comparison to continuous pasture and native Cerrado. During two years (2005 and 2006), soil samples from a clayed Rhodic Ferralsol were collected from two layers $(0-20$ and $70-80 \mathrm{~cm})$, in six areas under different crop rotation and ICL. Continuous pasture and native Cerrado were used as reference. The following physical and hydraulic properties were evaluated: bulk density, water saturation, total porosity, macroporosity, effective microporosity, effective saturation, lab and field-saturated hydraulic conductivity, and soil-water retention curve. All the cultivated systems caused impact on the physical and hydraulic properties of the $0-20 \mathrm{~cm}$ layer. The continuous pasture provided the best soil physical quality. In the comparison between years, only the soil under conventional tillage had its bulk density increased and its soil porosity decreased. The pasture in the rotation system with ICL, even after four years, does not restore soil physical quality in comparison to continuous pasture.
\end{abstract}

Index terms: hydraulic conductivity, retention curve, porosity, crop rotation, no-tillage system.

\section{Introdução}

A introdução de forrageiras perenes nos sistemas de produção de grãos, mesmo que manejados com rotação de culturas, pode trazer benefícios para a qualidade física do solo (Marchão et al., 2007). A degradação das pastagens é, atualmente, o principal passivo que compromete a sustentabilidade da pecuária, e pode ser explicada como um processo dinâmico de queda relativa da produtividade, principalmente em razão do manejo animal inadequado e da falta de reposição de nutrientes (Macedo, 2009). Os sistemas de integração lavoura-pecuária (ILP) podem ser alternativas para as dificuldades enfrentadas pela pecuária, na recuperação de pastagens degradadas, em virtude do maior aporte de resíduos vegetais e da melhoria da qualidade do solo (Vilela et al., 2008). Se for considerada a expressividade das áreas ocupadas sob pastagens no Brasil e, em especial, no Cerrado - 54,3 milhões de hectares sob pastagens cultivadas (Sano et al., 2008) -, a ILP exercerá um papel ainda mais importante para a sustentabilidade da agropecuária nessa região.

Os estudos sobre a qualidade física do solo evoluíram significativamente nos últimos anos, justificados quase sempre pela necessidade de se avaliar o comportamento de diversos atributos físicos do solo em áreas cultivadas

Pesq. agropec. bras., Brasília, v.46, n.10, p.1339-1348, out. 2011 
e sob pastagens (Lanzanova et al., 2007; Fidalski et al., 2008; Spera et al., 2009; Sales et al., 2010). No entanto, ainda são escassas as informações de experimentos de longa duração, em que seja possível observar o efeito acumulado das alterações provocadas pelos sistemas de manejo na qualidade física do solo.

Em experimento conduzido desde 1963, no Uruguai, Prechac (1992) analisou vários atributos físicos e observou que o solo degrada-se na lavoura contínua, mas recupera-se com a inclusão de pastagens de gramíneas em rotação. Esse autor relata que os benefícios da inclusão das pastagens na produção das culturas anuais dependem do manejo das pastagens, com melhores resultados em pastagens de alta produtividade.

Marchão et al. (2007), em experimento de longa duração na região do Cerrado, avaliaram a qualidade física do solo em sistemas de ILP, em comparação a sistemas tradicionais de cultivo e sistemas de plantio contínuo de lavoura e de pastagem, e constataram incremento na resistência à penetração e na densidade do solo por causa do pisoteio animal. Esses autores concluíram que, apesar de significativos, os impactos não foram limitantes à produção dos cultivos anuais subsequentes e também verificaram que os sistemas de ILP contribuíram para aumentar o armazenamento de água no solo, sobretudo quando associados ao sistema plantio direto (SPD).

Uma das limitações de estudos que visam demonstrar os benefícios de sistemas de ILP é a ausência do componente animal no sistema. Diversos trabalhos tratam apenas dos benefícios da inclusão de gramíneas, como plantas de cobertura na rotação, sem considerar que a presença dos animais exerce influência no sistema solo-planta-animal. As dificuldades para a realização de estudos que contemplam os três componentes do sistema estão relacionadas, principalmente, ao custo elevado para implantação de experimentos que representem as condições reais e as interações que ocorrem no sistema ILP, e ao tamanho das parcelas necessárias para comportar os animais, que nem sempre estão disponíveis em estações experimentais. Apesar do consenso de que sistemas de ILP condicionam melhorias na qualidade do solo e na produtividade de lavouras subsequentes, ainda não se sabe por quanto tempo esse sistema precisa permanecer na fase pastagem, para que os benefícios possam ser alcançados.

O objetivo deste trabalho foi avaliar a qualidade física do solo em sistemas de integração lavoura-pecuária, em comparação à pastagem contínua e ao cerrado nativo.

\section{Material e Métodos}

$\mathrm{O}$ experimento foi realizado na Embrapa Arroz e Feijão, Município de Santo Antônio de Goiás, GO, em Latossolo Vermelho Acriférrico típico, textura muito argilosa, A moderado, fase floresta tropical subcaducifólia e relevo plano (Santos et al., 2010). O clima, conforme a classificação de Köppen, é Aw, tropical de savana, megatérmico.

Os sistemas de manejo estudados compõem uma unidade em sistema ILP de aproximadamente 97 ha, em que foram avaliados sistemas agrícolas baseados na produção consorciada de grãos com forrageiras tropicais do gênero Urochloa (Syn. Brachiaria). Cada sistema compreendeu um piquete com área média de 13,5 ha, com pastejo durante todo o ano, na fase pastagem ou na entressafra, nos sistemas consorciados. O sistema de pastejo utilizado foi o rotacionado ( 9 dias de ocupação seguidos por 36 de descanso), com produtividade média anual de massa de matéria verde (MV, 74,5\% de umidade) das pastagens de Urochloa brizantha [(Hochst. ex A. Rich.) R.D. Webster] de 24,3 $\mathrm{Mg} \mathrm{ha}^{-1}$ de MV, em 11 avaliações de verão, em 2005 e 2006, segundo base de dados do Núcleo de Produção Animal da Embrapa Arroz e Feijão. A lotação média anual utilizada foi de 3,05 unidades animal por hectare (UA ha ${ }^{-1}$ ), com um ganho de peso de $700 \mathrm{~g} \mathrm{UA} \mathrm{ha}^{-1}$ por dia (Muniz, 2007).

Os seis sistemas de uso foram denominados de acordo com o uso na safra 2005/2006, como: P1, pastagem de $U$. brizantha, com quatro anos de uso; $\mathrm{P} 2$, pastagem de $U$. brizantha, com dois anos de uso; P3, pastagem de $U$. brizantha, com três anos de uso; $\mathrm{C} 1$, soja sob plantio direto; $\mathrm{C} 2$, arroz sob plantio convencional; e $\mathrm{C} 3$, milho consorciado com $U$. brizantha sob plantio direto. Como referências, foram coletadas amostras de solo em áreas com pastagem contínua, com 19 anos de implantação, e de cerrado nativo, adjacentes ao experimento. $\mathrm{Na}$ área de pastagem contínua (PC), a espécie utilizada foi Urochloa decumbens (Stapf) R.D. Webster, implantada em 1986. Essa área foi selecionada por apresentar baixa disponibilidade de forragem, decorrente do superpastejo, do pastejo contínuo durante o ano sem nenhum tipo de manejo, e sem qualquer tipo de adubação de manutenção. Os sistemas C1, C2 e C3, submetidos ao plantio de culturas anuais de inverno, foram irrigados por um pivô central.

Na Tabela 1, apresenta-se o histórico do uso do solo em cada área e sistema de manejo, desde a implantação dos sistemas ILP, em 1999. 
Em cada sistema, foram coletadas, no ponto médio de cada camada, amostras de solo em três trincheiras, dispostas em um transecto no sentido de maior comprimento do piquete, nas camadas de $0-20 \mathrm{~cm}$ e 70-80 cm. Em cada camada de solo, foram retiradas quatro amostras indeformadas, em anéis volumétricos de aço inoxidável de $5,1 \mathrm{~cm}$ de altura e $5,0 \mathrm{~cm}$ de diâmetro $\left(100 \mathrm{~cm}^{3}\right)$, que totalizaram 12 amostras por sistema. As amostras foram coletadas entre os meses de maio e julho de 2005 e 2006. Em 2006, a amostragem foi realizada apenas na camada de $0-20 \mathrm{~cm}$, para avaliar a evolução das alterações após um ano de rotação dos sistemas.

Em laboratório, as amostras indeformadas foram colocadas em uma bandeja com água a $2 / 3$ da altura do cilindro para saturação, durante 24 horas. Depois desse processo, foi avaliada a condutividade hidráulica saturada de laboratório $\left(\mathrm{K}_{\mathrm{SL}}\right)$, com uso do permeâmetro de carga constante (Claessen, 1997), em que as amostras foram submetidas a uma carga $\mathrm{H}=6,8 \mathrm{~cm}$. A $\mathrm{K}_{\mathrm{SL}}$ foi calculada por meio da seguinte equação, derivada da lei de Darcy: $K_{\mathrm{SL}}=\mathrm{Q} \times \mathrm{L} / \mathrm{A} \times \mathrm{T}$ x H, em que $\mathrm{K}_{\mathrm{SL}}$ é a condutividade hidráulica saturada de laboratório $\left(\mathrm{cm} \mathrm{s}^{-1}\right)$; Q é o volume de água $\left(\mathrm{cm}^{3}\right)$; L é a altura do cilindro $(\mathrm{cm})$; A é a área do cilindro $\left(\mathrm{cm}^{2}\right)$; $\mathrm{T}$ é o tempo (s); e H é a carga hidráulica $(\mathrm{cm})$.

Foram considerados por base os resultados de $\mathrm{K}_{\mathrm{SL}}$ obtidos nos 12 cilindros coletados. Selecionaram-se nove cilindros cujos valores de $\mathrm{K}_{\mathrm{SL}}$ foram considerados homogêneos e mais próximos da média para serem utilizados na determinação da curva de retenção de água (CRA) do solo. A CRA foi obtida pelo método da centrífuga (Silva \& Azevedo, 2002) e a umidade em equilíbrio foi determinada com os potenciais correspondentes a $-10,-30,-60,-100,-330,-800$, $-4.000,-10.000$, e -15.198,75 hPa (Reatto et al., 2008). Ao final do processo de dessorção da água, a densidade do solo $\left(\mathrm{D}_{\mathrm{s}}\right.$, em $\left.\mathrm{g} \mathrm{cm}^{-3}\right)$ foi mensurada pelo método do anel volumétrico, com massa do solo seco obtida na estufa a $105^{\circ} \mathrm{C}$. Para cada valor de umidade $(\theta)$, foi calculada a umidade relativa $\left(\theta_{\text {rel }}\right)$ com a fórmula,

$$
\theta_{\text {rel }}=\left(\theta-\theta_{15.198,75 \mathrm{hPa}}\right) /\left(\theta_{\mathrm{s}}-\theta_{15.198,75 \mathrm{hPa}}\right),
$$

em que: $\theta$ é a umidade gravimétrica correspondente a cada tensão; $\theta_{\mathrm{s}}$ é a umidade gravimétrica medida na saturação, determinada pela pesagem direta da amostra do solo saturado; $\theta_{15.198,75 \mathrm{hPa}}$ é a umidade gravimétrica correspondente ao potencial de $-15.198,75 \mathrm{hPa}$; e a diferença $\theta_{\mathrm{s}}-\theta_{15.198,75 \mathrm{hPa}}$ é a saturação efetiva (Se) de água no solo.

A CRA foi ajustada ao modelo de van Genuchten (Genuchten, 1980), adaptado por Santos (2010), de acordo com a equação: $\theta_{\text {rel }}=\left\{\theta_{\mathrm{r}}+\left[\theta_{\mathrm{s}}-\theta_{\mathrm{r}}\right][1+(\alpha \ln \right.$ $\left.\left.\mathrm{T})^{\mathrm{n}}\right]^{-\mathrm{m}}-\theta_{15.198,75}\right\} /\left(\theta_{\mathrm{s}}-\theta_{15.198,75}\right)$. Os parâmetros de ajuste $\left(\theta_{\mathrm{r}}, \alpha, \mathrm{n}\right.$ e $\left.\mathrm{m}\right)$ do modelo foram obtidos por meio da rotina "Solver" do programa Microsoft Excel, em que $\theta_{\text {rel }}$ é a umidade gravimétrica relativa $\left(\mathrm{g} \mathrm{g}^{-1}\right)$; $\theta_{\mathrm{s}}$ é a umidade gravimétrica na saturação $\left(\mathrm{g} \mathrm{g}^{-1}\right)$; $\theta_{\mathrm{r}}$ é a umidade gravimétrica residual do solo $\left(\mathrm{g} \mathrm{g}^{-1}\right)$, ajustada ao potencial de $-15.198,75 \mathrm{hPa} ; \ln \mathrm{T}$ é o potencial mátrico na escala $\ln \mathrm{T}(\ln \mathrm{hPa}) ; \alpha$ é o parâmetro de escala; $m$ e $n$ são parâmetros de forma, adimensionais; $\theta_{15.198,75}$ é a umidade gravimétrica medida $\left(\mathrm{g} \mathrm{g}^{-1}\right)$ no potencial mátrico de $-15.198,75 \mathrm{hPa}$.

Tabela 1. Sistemas de manejo e uso do solo em integração lavoura-pecuária.

\begin{tabular}{|c|c|}
\hline Sistemas de manejo & Sequência anual das culturas (verão/inverno) no período 1999-2006 \\
\hline P1 & $\begin{array}{l}\text { Pousio - soja/pousio - soja/pousio - soja/pousio - milho }+U . \text { brizantha/U. brizantha }-U . \text { brizantha/U. brizantha }-U . \text { brizantha/ } \\
\text { U. brizantha }-U . \text { brizantha }\end{array}$ \\
\hline $\mathrm{P} 2$ & $\begin{array}{l}\text { Pousio - milho/pousio - milho }+U . \text { brizantha/U. brizantha }- \text { soja }+U . \text { brizantha } / \text { milho }- \text { milho }+U . \text { brizantha/U. brizantha }- \text { arroz/ } \\
\text { feijão }- \text { soja }+U . \text { brizantha/U. brizantha }-U . \text { brizantha }\end{array}$ \\
\hline P3 & $\begin{array}{l}\text { Pousio }- \text { milho/milheto }- \text { soja }+U . \text { brizantha } / U . \text { brizantha }- \text { milho }+U . \text { brizantha } U . \text { brizantha }- \text { soja } / \text { milho }- \text { milho }+U . \text { brizantha } \\
U . \text { brizantha }-U . \text { brizantha/U. brizantha }-U . \text { brizantha }\end{array}$ \\
\hline $\mathrm{C} 1$ & $\begin{array}{l}\text { Feijão }- \text { milho/feijão }- \text { soja }^{(1)} / \text { cevada }^{(1)}-\text { milho }+U . \text { brizantha/U. brizantha }-U . \text { brizantha/U. brizantha }-U . \text { brizantha/U. brizantha } \\
-U . \text { brizantha/U. brizantha }- \text { soja }\end{array}$ \\
\hline $\mathrm{C} 2$ & $\begin{array}{l}\text { Feijão }- \text { milho/feijão }- \text { soja }^{(1)} / \text { cevada }- \text { milho }+U . \text { brizantha/U. brizantha }-U . \text { brizantha/U. brizantha }-U . \text { brizantha/U. brizantha }- \\
\text { soja/feijão }{ }^{(1)}-\text { arroz }^{(1)}\end{array}$ \\
\hline $\mathrm{C} 3$ & 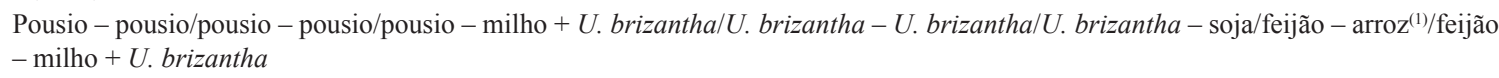 \\
\hline Pastagem contínua & U. decumbens, com 19 anos de implantação, sem qualquer forma de manejo \\
\hline Cerrado nativo & - \\
\hline
\end{tabular}

\footnotetext{
${ }^{(1)}$ Áreas cultivadas sob preparo convencional do solo, com grade aradora.
} 
Para cada uma das áreas e em cada camada de solo, foi construída uma CRA, o que resultou em 16 curvas. Todas as curvas foram comparadas entre si, por camada de solo, pelo procedimento descrito por Silva \& Azevedo (2002), também utilizado por Marchão et al. (2007), ao se considerar a soma de quadrados dos erros (SQ erro $_{\text {) }}$ obtidos no ajuste da CRA. A partir dessa comparação, foi possível determinar um valor de F, com 3 graus de liberdade no numerador e 10 no denominador (Silva \& Azevedo, 2002), a 5\% de probabilidade, correspondente a cada uma das 28 comparações realizadas entre os sistemas de manejo e entre as camadas avaliadas.

$\mathrm{O}$ atributo macroporosidade ( $\mathrm{Ma}$, em $\mathrm{g} \mathrm{g}^{-1}$ ) foi calculado como a diferença entre a porosidade total do solo (PT, em g g$^{-1}$ ) (Silva \& Azevedo, 2001) e as respectivas umidades em equilíbrio com o potencial de $-60 \mathrm{hPa}$, de acordo com a equação $\mathrm{Ma}=\mathrm{PT}-\theta_{60}$, em que $\theta_{60}$ é a umidade gravimétrica medida no potencial de $-60 \mathrm{hPa}$, e PT é a porosidade total $\left(\mathrm{g} \mathrm{g}^{-1}\right)$. O potencial de $-60 \mathrm{hPa}$ foi considerado como o limite de referência entre a macroporosidade e a microporosidade (Mi, em $\mathrm{g} \mathrm{g}^{-1}$ ), obtida em cada CRA. A microporosidade efetiva $\left(\mathrm{Mi}_{\mathrm{e}}\right)$ foi calculada mediante a equação $\mathrm{Mi}_{\mathrm{e}}=\theta_{60}-\theta_{15.198,75}$.

A densidade de partículas do solo $\left(\mathrm{D}_{\mathrm{p}}\right.$, em $\left.\mathrm{g} \mathrm{cm}^{-3}\right)$ foi calculada por meio da equação $\mathrm{D}_{\mathrm{p}}=\mathrm{Ms} / \mathrm{Vs}$ (Claessen, 1997), em que Ms é a massa do solo seco em estufa a $105^{\circ} \mathrm{C}(\mathrm{em} \mathrm{g}) ; \mathrm{Vs}$ é o volume original do solo seco da amostra $\left(\mathrm{cm}^{3}\right)$, medido antes da centrifugação.

A porosidade total do solo (PT, em $\mathrm{g} \mathrm{g}^{-1}$ ) ou volume total de poros foi calculada mediante a equação $\mathrm{PT}=1 / \mathrm{D}_{\mathrm{s}}-1 / \mathrm{D}_{\mathrm{p}}$, em que $\mathrm{D}_{\mathrm{s}}$ é a densidade do solo $\left(\mathrm{g} \mathrm{cm}^{-3}\right)$ e $\mathrm{D}_{\mathrm{p}}$ é a densidade de partículas do solo $\left(\mathrm{g} \mathrm{cm}^{-3}\right)$.

A condutividade hidráulica saturada de campo $\left(\mathrm{K}_{\mathrm{SG}}\right)$ foi avaliada com o uso do permeâmetro de Guelph (Reynolds \& Elrick, 1987), a partir de furos no solo com um raio $(\mathrm{R})$ de $3 \mathrm{~cm}$, e carga hidráulica $(\mathrm{H})$ de $3 \mathrm{ou}$ $5 \mathrm{~cm}$, o que dependeu da velocidade de infiltração da água no solo. Foram realizadas três medições, cada uma nos mesmos pontos em que foram abertas as trincheiras, o que totalizou nove repetições por camada em cada sistema (0-20 e 70-80 cm, para 2005, e 0-20 cm para 2006). Os valores de $K_{S G}$ foram obtidos pela equação, $\mathrm{K}_{\mathrm{SG}}=\mathrm{CQ} /\left(2 \pi \mathrm{H}^{2}+\mathrm{C} \pi \mathrm{R}^{2}+2 \pi \mathrm{H} / \mathrm{A}\right)$, de Reynolds \& Elrick (1987), em que $\mathrm{K}_{\mathrm{SG}}$ é a condutividade hidráulica saturada de campo $\left(\mathrm{cm} \mathrm{s}^{-1}\right)$; Q é a vazão média da água infiltrada no solo $\left(\mathrm{m}^{3} \mathrm{~s}^{-1}\right)$; H é a carga hidráulica $(\mathrm{cm})$; $\mathrm{R}$ é o raio do furo $(\mathrm{cm})$; A é o parâmetro associado à textura e à estrutura do solo $\left(\mathrm{cm}^{-1}\right) ; \mathrm{C}$ é um fator adimensional, estabelecido em função da relação H/R.

Para as comparações entre os atributos físico-hídricos na camada 0-20 cm, em cada sistema de manejo e para cada ano, foram organizados modelos gráficos do tipo diagrama radial, em que os atributos físico-hídricos foram dispostos em um dos raios para cada sistema de manejo. A pastagem contínua foi considerada como área de referência, já que o objetivo da análise gráfica foi comparar os sistemas cultivados. Os dados foram transformados ao considerar-se 1,0 para os valores da pastagem contínua. Foi calculado o quociente entre o valor obtido do atributo físico-hídrico e o valor de referência. Quando o resultado referia-se a um atributo físico-hídrico que deveria ser menor do que o de referência - densidade do solo e microporosidade efetiva, por exemplo -, utilizou-se o inverso do valor determinado no cálculo.

Para comparação das médias dos atributos físico-hídricos, foi utilizado o teste t de Student, a 5\% de probabilidade. Os gráficos das curvas de retenção de água e os diagramas de QFS foram construídos com o programa Microsoft Excel.

\section{Resultados e Discussão}

Os sistemas de manejo com ILP apresentaram redução na qualidade física do solo em relação à área de cerrado (Tabela 2 e Figura 1). Na camada mais profunda, não houve efeito dos sistemas de manejo, que apresentaram valores muito semelhantes para a maioria dos atributos físico-hídricos avaliados. Entre os atributos avaliados, os únicos que apresentaram diferenças entre os sistemas, na camada $70-80 \mathrm{~cm}$, foram a macroporosidade $\mathrm{e}$ as condutividades hidráulicas saturadas de campo e de laboratório. Esses atributos estão diretamente ligados à estrutura do solo e podem ter apresentado essas variações em virtude de alterações de ordem biológica decorrentes dos sistemas de uso, como, por exemplo, a presença de bioporos. Reatto et al. (2009) demonstraram que a evolução da estrutura de Latossolos do Planalto Central é resultante da atividade biológica, principalmente da macrofauna edáfica.

Os maiores valores de densidade do solo na camada de 0-20 cm de profundidade foram observados nos sistemas C3, C1, P3, P2 e P1, que apresentaram incrementos de $31,4,30,5,27,6,23,8$ e $23,8 \%$, respectivamente, em comparação ao cerrado. Além do incremento nos valores 
de densidade, observou-se decréscimo nos valores de macroporosidade nessas áreas.
Os maiores valores de densidade do solo observados na camada superficial são indicação de que o uso

Tabela 2. Valores médios de atributos físico-hídricos (2005), de acordo com sistemas de manejo do solo e com a profundidade ${ }^{(1)}$.

\begin{tabular}{|c|c|c|c|c|c|c|c|c|c|}
\hline \multirow[t]{2}{*}{ Atributos $^{(2)}$} & \multirow{2}{*}{$\begin{array}{c}\text { Camada } \\
(\mathrm{cm})\end{array}$} & \multicolumn{8}{|c|}{ Sistemas de uso e manejo $^{(3)}$} \\
\hline & & $\mathrm{CE}$ & $\mathrm{PC}$ & $\mathrm{P} 1$ & $\mathrm{P} 2$ & P3 & $\mathrm{C} 1$ & $\mathrm{C} 2$ & $\mathrm{C} 3$ \\
\hline \multirow{2}{*}{$\mathrm{D}_{\mathrm{s}}\left(\mathrm{g} \mathrm{cm}^{-3}\right)$} & $0-20$ & $1,05 \mathrm{~d}$ & $1,21 \mathrm{c}$ & $1,30 \mathrm{abc}$ & $1,30 \mathrm{abc}$ & $1,34 \mathrm{ab}$ & $1,37 \mathrm{ab}$ & $1,26 \mathrm{bc}$ & $1,38 \mathrm{a}$ \\
\hline & $70-80$ & $1,09 \mathrm{a}$ & $1,13 \mathrm{a}$ & $1,16 \mathrm{a}$ & $1,09 a$ & $1,21 \mathrm{a}$ & $1,22 \mathrm{a}$ & $1,11 \mathrm{a}$ & $1,18 \mathrm{a}$ \\
\hline \multirow{2}{*}{$\theta_{\mathrm{s}}\left(\mathrm{g} \mathrm{g}^{-1}\right)$} & $0-20$ & $0,54 \mathrm{a}$ & $0,43 b$ & $0,37 \mathrm{bcd}$ & $0,37 \mathrm{bcd}$ & $0,36 \mathrm{~cd}$ & $0,34 \mathrm{~cd}$ & $0,39 b c$ & $0,31 d$ \\
\hline & $70-80$ & $0,53 \mathrm{a}$ & $0,49 \mathrm{a}$ & $0,46 a$ & $0,51 \mathrm{a}$ & $0,42 \mathrm{a}$ & $0,41 \mathrm{a}$ & $0,48 \mathrm{a}$ & $0,44 \mathrm{a}$ \\
\hline \multirow{2}{*}{ PT $\left(\mathrm{g} \mathrm{g}^{-1}\right)$} & $0-20$ & $0,60 \mathrm{a}$ & $0,47 b$ & $0,40 \mathrm{bc}$ & $0,40 \mathrm{bc}$ & $0,39 \mathrm{bc}$ & $0,36 \mathrm{c}$ & $0,43 \mathrm{bc}$ & $0,36 \mathrm{c}$ \\
\hline & $70-80$ & $0,60 \mathrm{a}$ & $0,54 \mathrm{a}$ & $0,50 \mathrm{a}$ & $0,57 \mathrm{a}$ & $0,48 \mathrm{a}$ & $0,46 \mathrm{a}$ & $0,55 \mathrm{a}$ & $0,48 \mathrm{a}$ \\
\hline \multirow{2}{*}{$\mathrm{Ma}\left(\mathrm{g} \mathrm{g}^{-1}\right)$} & $0-20$ & $0,28 \mathrm{a}$ & $0,12 b c$ & $0,09 b c$ & $0,09 b c$ & $0,10 b c$ & $0,08 \mathrm{c}$ & $0,16 b$ & $0,10 b c$ \\
\hline & $70-80$ & $0,29 a$ & $0,22 \mathrm{ab}$ & $0,17 b$ & $0,22 \mathrm{ab}$ & $0,15 b$ & $0,14 \mathrm{~b}$ & $0,22 \mathrm{ab}$ & $0,18 \mathrm{ab}$ \\
\hline \multirow{2}{*}{$\mathrm{Mi}_{\mathrm{e}}\left(\mathrm{g} \mathrm{g}^{-1}\right)$} & $0-20$ & $0,33 \mathrm{ab}$ & $0,35 \mathrm{a}$ & $0,31 \mathrm{bc}$ & $0,31 b c$ & $0,29 \mathrm{~cd}$ & $0,28 \mathrm{~cd}$ & $0,27 \mathrm{~d}$ & $0,27 d$ \\
\hline & $70-80$ & $0,31 \mathrm{a}$ & $0,32 \mathrm{a}$ & $0,33 \mathrm{a}$ & $0,35 \mathrm{a}$ & $0,33 \mathrm{a}$ & $0,32 \mathrm{a}$ & $0,32 \mathrm{a}$ & $0,30 \mathrm{a}$ \\
\hline \multirow{2}{*}{$\mathrm{S}_{\mathrm{e}}\left(\mathrm{g} \mathrm{g}^{-1}\right)$} & $0-20$ & $0,32 \mathrm{a}$ & $0,20 \mathrm{~b}$ & $0,17 \mathrm{bc}$ & $0,16 b c$ & $0,17 \mathrm{bc}$ & $0,14 \mathrm{c}$ & $0,21 b$ & $0,13 \mathrm{c}$ \\
\hline & $70-80$ & $0,35 \mathrm{a}$ & $0,30 \mathrm{a}$ & $0,25 \mathrm{a}$ & $0,31 \mathrm{a}$ & $0,24 \mathrm{a}$ & $0,22 \mathrm{a}$ & $0,29 \mathrm{a}$ & $0,25 \mathrm{a}$ \\
\hline \multirow{2}{*}{$\mathrm{K}_{\mathrm{SL}}\left(\mathrm{cm} \mathrm{s}^{-1}\right)$} & $0-20$ & $102,98 \mathrm{a}$ & $15,89 b$ & $9,17 b$ & $2,01 b$ & $2,77 b$ & $3,05 b$ & $28,49 b$ & $4,49 b$ \\
\hline & $70-80$ & $67,65 a$ & $38,52 \mathrm{bc}$ & $7,06 \mathrm{~d}$ & $14,11 \mathrm{~cd}$ & $6,63 d$ & $11,30 \mathrm{~cd}$ & $61,22 \mathrm{ab}$ & $26,72 \mathrm{~cd}$ \\
\hline \multirow{2}{*}{$\mathrm{K}_{\mathrm{SG}}\left(\mathrm{cm} \mathrm{s}^{-1}\right)$} & $0-20$ & $8,54 \mathrm{ab}$ & $11,06 a$ & $2,48 \mathrm{~d}$ & $3,89 \mathrm{~cd}$ & $3,57 \mathrm{~d}$ & $3,86 \mathrm{~cd}$ & $6,71 b c$ & $1,84 d$ \\
\hline & $70-80$ & $21,38 \mathrm{ab}$ & $23,67 \mathrm{a}$ & $13,38 \mathrm{c}$ & $11,72 \mathrm{c}$ & $16,35 \mathrm{bc}$ & $15,42 \mathrm{bc}$ & $10,76 \mathrm{c}$ & $13,03 \mathrm{c}$ \\
\hline
\end{tabular}

${ }^{(1)}$ Médias seguidas de letras iguais, nas linhas, não diferem entre si pelo teste de t, a $5 \%$ de probabilidade. ${ }^{(2)} \mathrm{D}_{\mathrm{s}}$, densidade do solo; $\theta_{\mathrm{s}}$, umidade gravimétrica do solo na amostragem; PT, porosidade total do solo; Ma, macroporosidade; $\mathrm{Mi}_{\mathrm{e}}$, microporosidade efetiva; $\mathrm{S}_{\mathrm{e}}$, saturação efetiva; $\mathrm{K}_{\mathrm{SL}}$, condutividade hidráulica saturada de laboratório; $\mathrm{K}_{\mathrm{SG}}$, condutividade hidráulica saturada de campo. ${ }^{\left({ }^{3}\right)} \mathrm{CE}$, cerrado nativo; $\mathrm{PC}$, pastagem contínua, com 19 anos de uso; P1, pastagem de Urochloa brizantha, com quatro anos de uso; P2, pastagem de $U$. brizantha, com dois anos de uso; P3, pastagem de $U$. brizantha, com três anos de uso; $\mathrm{C} 1$, soja sob plantio direto; $\mathrm{C} 2$, arroz em plantio convencional; $\mathrm{C} 3, \mathrm{U}$. brizantha em consórcio com milho.

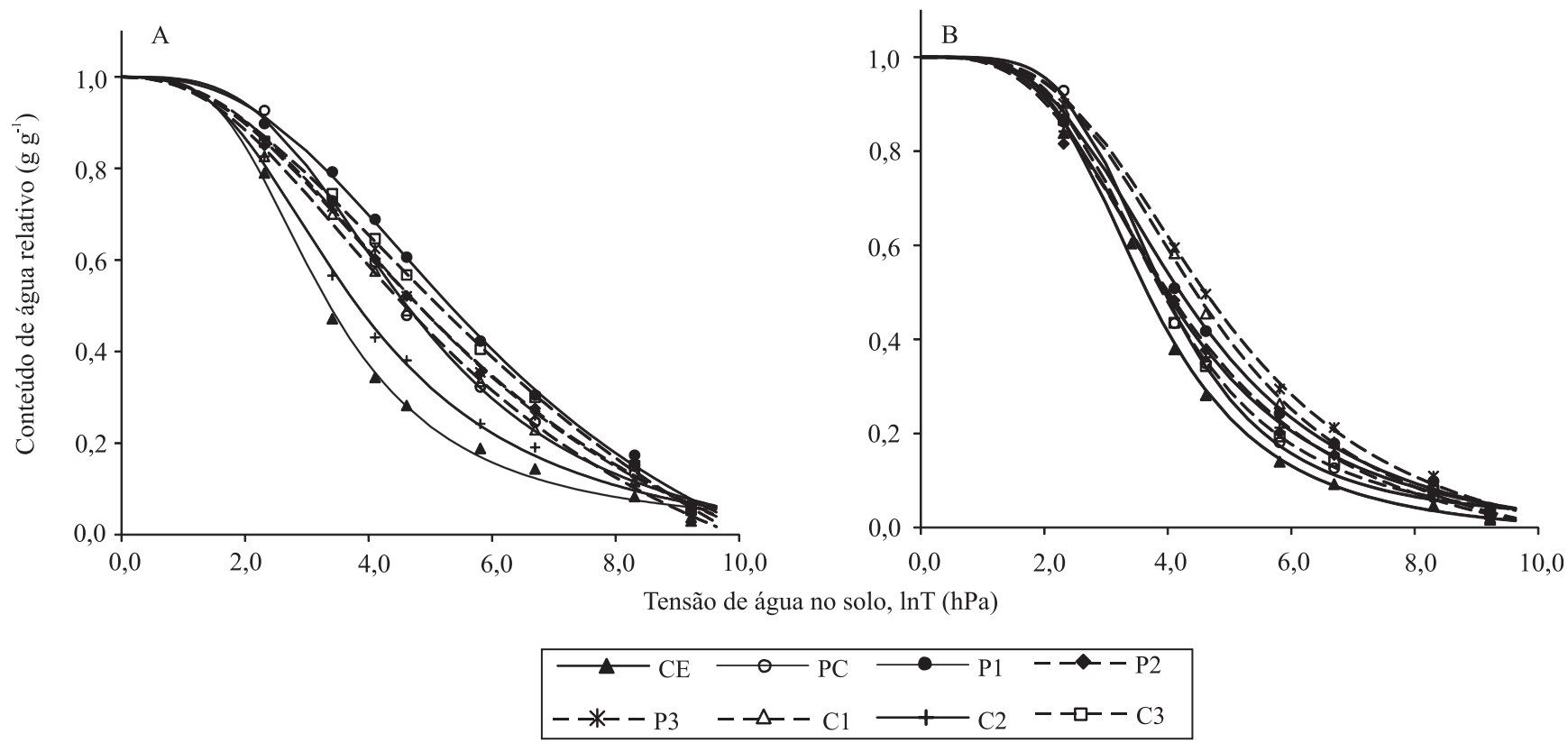

Figura 1. Curvas de retenção de água no solo (CRA) versus tensão da água no solo, com valores ajustados (aju) e observados (obs), em sistemas de integração lavoura-pecuária, nas camadas de 0-20(A) e 70-80 cm (B). CE, cerrado nativo; PC, pastagem contínua, com 19 anos de uso; P1, pastagem de Urochloa brizantha, com quatro anos de uso; P2, pastagem de $U$. brizantha, com dois anos de uso; P3, pastagem de $U$. brizantha, com três anos de uso; C1, soja sob plantio direto; $\mathrm{C} 2$, arroz em plantio convencional; $\mathrm{C} 3$, milho consorciado com $U$. brizantha sob plantio direto. 
intensivo do solo, com dois cultivos anuais, promoveu a sua compactação. Mesmo nos sistemas C1, P1, P2 e P3, com maior permanência da pastagem no sistema de rotação, houve incremento significativo na densidade do solo. $\mathrm{O}$ valor considerado elevado para a $\mathrm{D}_{\mathrm{s}}$, observado no sistema $\mathrm{C} 1$, pode estar associado ao fato de que, antes da implantação da cultura da soja na safra 2005/2006, a área permaneceu sob pastagem com altas taxas de lotação por um período de quatro anos (Tabela 1). Mesmo com pastejo rotacionado e manejo adequado da pastagem, a taxa de lotação em sistemas intensivos de pastejo influencia a magnitude da compactação provocada pelo pisoteio, que parece ter sido a principal contribuição para o incremento da $\mathrm{D}_{\mathrm{s}}$. Com exceção da pastagem contínua, todas as outras áreas de pastagens eram de U. brizantha, espécie que apresenta hábito de crescimento cespitoso, o que pode favorecer a compactação do solo nas áreas entre touceiras. Marchão et al. (2007) também observaram aumento de densidade no perfil do solo em pastagens e plantio direto, principalmente quando utilizaram forrageiras com hábito de crescimento cespitoso.

Em geral, os sistemas de manejo apresentaram perda de porosidade em comparação ao cerrado, o que foi evidenciado pela análise dos atributos PT, $\mathrm{Ma}$ e $\mathrm{Mi}_{\mathrm{e}}$. Destacam-se, em posição intermediária, a pastagem contínua e o sistema $\mathrm{C} 2$. No caso da pastagem contínua, embora tenha havido incremento na $\mathrm{D}_{\mathrm{s}}$, a redução nos valores dos atributos ligados à porosidade não foi tão acentuada quanto nos sistemas de manejo em rotação, conforme observado nos sistemas $\mathrm{C} 1$ e $\mathrm{C} 3$, que apresentaram os menores valores de PT e Ma. A manutenção da porosidade do solo na área sob pastagem contínua ocorreu, provavelmente, por não ter havido revolvimento contínuo do solo, bem como em razão do sistema radicular da gramínea e de invertebrados edáficos, que podem ter contribuído para melhoria da sua estruturação física. Ao comparar-se o sistema C2 com os outros sistemas de manejo, observaram-se valores intermediários de PT e Ma. É provável que esse resultado tenha decorrido do fato de que, nos dois últimos cultivos das culturas de feijão e arroz, o solo dessa área foi preparado com grade aradora.

Segundo Spera et al. (2009), a compactação remanescente de mobilizações do solo pela aração e gradagem praticadas antes da adoção do sistema plantio direto, para implantação da ILP, podem ser notadas por um longo período no solo. Lanzanova et al. (2007) avaliaram atributos físicos do solo em sistema ILP e verificaram que a compactação decorrente do pisoteio bovino, durante três anos de avaliações, limitou-se à camada $0-10 \mathrm{~cm}$ de solo. De acordo com Magalhães et al. (2001), houve aumento progressivo, ao longo dos anos, na $\mathrm{D}_{\mathrm{s}} \mathrm{da}$ camada $0-10 \mathrm{~cm}$ em razão do pastejo; contudo, esse incremento na densidade não prejudicou a produção de massa de matéria verde de $U$. brizantha. Marchão et al. (2009) demonstraram que a compactação superficial do solo, pelo pisoteio animal, é facilmente reversível, e pode ser rompida por semeadoras recomendadas para o sistema plantio direto.

$\mathrm{AK}_{\mathrm{SL}}$ na camada $0-20 \mathrm{~cm}$ diminuiu em comparação à área de cerrado, em todos os sistemas, inclusive na área de pastagem contínua. No entanto, ao se comparar os sistemas que utilizaram a $\mathrm{K}_{\mathrm{SG}}$, medida pelo permeâmetro de Guelph, a pastagem contínua apresentou valores superiores a todos os outros sistemas, exceto o cerrado. O sistema $\mathrm{C} 3$ foi o que apresentou o menor valor do atributo $\mathrm{K}_{\mathrm{SG}}$, o que demonstra que o manejo adotado pode ter comprometido o fluxo da água no perfil do solo e, consequentemente, a infiltração. Silva \& Kato (1997) compararam sistema de plantio direto e convencional em Latossolo e demonstraram que, em solo descoberto ou desestruturado pelo preparo, pode ocorrer redução da condutividade hidráulica, como consequência do selamento superficial do solo. Albuquerque et al. (2001) avaliaram o comportamento de atributos físico-hídricos em um Nitossolo em sistemas ILP e encontraram resultados semelhantes. Os autores concluíram que a compactação imposta pela ILP reduziu a macroporosidade e a condutividade hidráulica saturada, além de ter aumentado a resistência do solo à penetração.

Ao se avaliar a $\mathrm{K}_{\mathrm{SL}}$ na camada de $70-80 \mathrm{~cm}$, os valores obtidos para o sistema PC foram superiores a todos os sistemas de cultivo. Os valores medidos de $K_{\mathrm{SG}}$ na pastagem para essa camada de solo foram superiores, inclusive na área de vegetação nativa. O efeito do sistema radicular da vegetação nativa e da pastagem, e a atividade da macrofauna, por meio da construção de galerias e bioporos, pode influenciar a condutividade hidráulica de campo $\left(\mathrm{K}_{\mathrm{SG}}\right)$, o que faz com que esses resultados apresentem 
alta variabilidade. A condutividade hidráulica de laboratório $\left(\mathrm{K}_{\mathrm{SL}}\right)$ é medida em amostras indeformadas de solo, que estão sujeitas a uma desestruturação no ato da coleta, conforme observaram Mesquita \& Morais (2004). Os autores destacam que os resultados de condutividade hidráulica e macroporosidade são provenientes do mesmo espaço poroso, portanto, é de se esperar que haja correlação entre essas duas variáveis.

Em todos os sistemas de ILP, a $\theta_{\mathrm{s}}, \mathrm{Mi}_{\mathrm{e}}$ e a $\mathrm{S}_{\mathrm{e}}$ foram afetadas pelos sistemas de manejo, em comparação ao cerrado, exceto no caso da $\mathrm{Mi}_{\mathrm{e}}$ na pastagem contínua, que apresentou valor semelhante ao observado no cerrado (Tabela 2). Essa exceção também foi observada em relação à condutividade da água no solo, e é explicada pela provável ausência de selamento superficial, em virtude da presença permanente de cobertura dos resíduos na superfície do solo e do agressivo sistema radicular das gramíneas. Broch (2000) estudou a quantidade e a distribuição do sistema radicular em uma pastagem de $U$. decumbens e observou que a gramínea apresentou $1,741 \mathrm{Mg} \mathrm{ha}^{-1}$ de massa de matéria seca de raízes até $100 \mathrm{~cm}$ de profundidade; assim, há influência do sistema radicular sobre os atributos físico-hídricos do solo.
Ao se compararem os resultados das amostragens nos sistemas agrícolas entre os dois anos de avaliação (Tabela 3), observaram-se alterações significativas somente no sistema $\mathrm{C} 2$, em que houve preparo convencional do solo. Neste sistema, houve incremento significativo na $\mathrm{D}_{\mathrm{s}}$ e redução dos atributos $\theta_{\mathrm{s}}$, PT, Ma, $\mathrm{S}_{\mathrm{e}}$ e $\mathrm{K}_{\mathrm{sG}}$, o que demonstra que o preparo convencional contínuo pode reduzir a porosidade e provocar a compactação do solo. $\mathrm{Na}$ área de cerrado e na pastagem, houve diferença significativa nos atributos $\mathrm{Mi}_{\mathrm{e}}$ e $\mathrm{K}_{\mathrm{SG}}$, o que indica que nesses sistemas há uma grande variabilidade espacial desses atributos.

$\mathrm{Na}$ camada superficial, todas as CRA dos sistemas de manejo foram significativamente diferentes da CRA do cerrado (Figura 1 e Tabela 4). O sistema C2 foi o que apresentou a CRA mais semelhante à do cerrado. O preparo do solo produziu efeito positivo sobre a estrutura na camada superficial. Entretanto, os resultados dos outros atributos físico-hídricos demonstraram que o preparo do solo por dois anos consecutivos prejudicou a qualidade física do solo. Houve tendência de aproximação das curvas com o aumento da profundidade $(70-80 \mathrm{~cm})$. Com exceção do sistema P3,

Tabela 3. Valores médios de atributos físico-hídricos para os sistemas de uso e manejo do solo na camada 0-20 cm para 2005 e $2006^{(1)}$.

\begin{tabular}{|c|c|c|c|c|c|c|c|c|c|}
\hline \multirow[t]{2}{*}{ Atributos $^{(2)}$} & \multirow[t]{2}{*}{ Anos } & \multicolumn{8}{|c|}{ Sistemas de uso e manejo $^{(3)}$} \\
\hline & & $\mathrm{CE}$ & $\mathrm{PC}$ & P1 & $\mathrm{P} 2$ & P3 & $\mathrm{C} 1$ & $\mathrm{C} 2$ & $\mathrm{C} 3$ \\
\hline \multirow{2}{*}{$\mathrm{D}_{\mathrm{s}}\left(\mathrm{g} \mathrm{cm}^{-3}\right)$} & 2005 & $1,05 \mathrm{dA}$ & $1,21 \mathrm{cA}$ & $1,30 \mathrm{abcA}$ & $1,30 \mathrm{abcA}$ & $1,34 \mathrm{abA}$ & $1,37 \mathrm{abA}$ & $1,26 \mathrm{bcB}$ & $1,38 \mathrm{aA}$ \\
\hline & 2006 & $1,07 \mathrm{cA}$ & $1,27 \mathrm{bA}$ & $1,26 \mathrm{bA}$ & $1,32 \mathrm{bA}$ & $1,41 \mathrm{aA}$ & $1,40 \mathrm{aA}$ & $1,44 \mathrm{aA}$ & $1,31 \mathrm{bA}$ \\
\hline \multirow{2}{*}{$\theta_{\mathrm{s}}\left(\mathrm{g} \mathrm{g}^{-1}\right)$} & 2005 & $0,54 \mathrm{aA}$ & $0,43 \mathrm{bA}$ & $0,37 \mathrm{bcdA}$ & $0,37 \mathrm{bcdA}$ & $0,36 \mathrm{cdA}$ & $0,34 \mathrm{cdA}$ & $0,39 \mathrm{bcA}$ & $0,31 \mathrm{dA}$ \\
\hline & 2006 & $0,50 \mathrm{aA}$ & $0,38 \mathrm{bA}$ & $0,39 \mathrm{bA}$ & $0,36 \mathrm{cA}$ & $0,31 \mathrm{efA}$ & $0,33 \mathrm{deA}$ & $0,30 \mathrm{fB}$ & $0,35 \mathrm{cdA}$ \\
\hline \multirow{2}{*}{ PT $\left(\mathrm{g} \mathrm{g}^{-1}\right)$} & 2005 & $0,60 \mathrm{aA}$ & $0,47 \mathrm{bA}$ & $0,40 \mathrm{bcA}$ & $0,40 \mathrm{bcA}$ & $0,39 \mathrm{bcA}$ & $0,36 \mathrm{cA}$ & $0,43 \mathrm{bcA}$ & $0,36 \mathrm{cA}$ \\
\hline & 2006 & $0,58 \mathrm{aA}$ & $0,43 \mathrm{bA}$ & $0,43 \mathrm{bA}$ & $0,39 \mathrm{bcA}$ & $0,36 \mathrm{cdA}$ & $0,35 \mathrm{dA}$ & $0,32 \mathrm{~dB}$ & $0,41 \mathrm{bA}$ \\
\hline \multirow{2}{*}{$\mathrm{Ma}\left(\mathrm{g} \mathrm{g}^{-1}\right)$} & 2005 & $0,28 \mathrm{aA}$ & $0,12 \mathrm{bcA}$ & $0,09 \mathrm{bcA}$ & $0,09 \mathrm{bcA}$ & $0,10 \mathrm{bcA}$ & $0,08 \mathrm{cA}$ & $0,16 \mathrm{bA}$ & $0,10 \mathrm{bcA}$ \\
\hline & 2006 & $0,25 \mathrm{aA}$ & $0,12 \mathrm{bA}$ & $0,11 \mathrm{bcA}$ & $0,10 \mathrm{bcA}$ & $0,09 \mathrm{bcdA}$ & $0,08 \mathrm{cdA}$ & $0,06 \mathrm{~dB}$ & $0,13 \mathrm{bA}$ \\
\hline \multirow{2}{*}{$\mathrm{Mi}_{\mathrm{e}}\left(\mathrm{g} \mathrm{g}^{-1}\right)$} & 2005 & $0,33 \mathrm{abB}$ & $0,35 \mathrm{aA}$ & $0,31 \mathrm{bcA}$ & $0,31 \mathrm{bcA}$ & $0,29 \mathrm{cdA}$ & $0,28 \mathrm{cdA}$ & $0,27 \mathrm{dA}$ & $0,27 \mathrm{dA}$ \\
\hline & 2006 & $0,36 \mathrm{aA}$ & $0,31 \mathrm{cB}$ & $0,32 \mathrm{bA}$ & $0,29 \mathrm{dA}$ & $0,27 \mathrm{fA}$ & $0,27 \mathrm{fA}$ & $0,26 \mathrm{fA}$ & $0,28 \mathrm{eA}$ \\
\hline \multirow{2}{*}{$\mathrm{S}_{\mathrm{e}}\left(\mathrm{g} \mathrm{g}^{-1}\right)$} & 2005 & $0,32 \mathrm{aA}$ & $0,20 \mathrm{bA}$ & $0,17 \mathrm{bcA}$ & $0,16 \mathrm{bcA}$ & $0,17 \mathrm{bcA}$ & $0,14 \mathrm{cA}$ & $0,21 \mathrm{bA}$ & $0,13 \mathrm{cA}$ \\
\hline & 2006 & $0,28 \mathrm{aA}$ & $0,20 \mathrm{bA}$ & $0,17 \mathrm{cdA}$ & $0,16 \mathrm{cdeA}$ & $0,14 \mathrm{deA}$ & $0,13 \mathrm{efA}$ & $0,12 \mathrm{fB}$ & $0,18 \mathrm{cA}$ \\
\hline \multirow{2}{*}{$\mathrm{K}_{\mathrm{SL}}\left(\mathrm{cm} \mathrm{s}^{-1}\right)$} & 2005 & $102,98 \mathrm{aA}$ & $15,89 \mathrm{bA}$ & $9,17 \mathrm{bA}$ & $2,01 \mathrm{bA}$ & $2,77 \mathrm{bA}$ & $3,05 \mathrm{bA}$ & $28,49 \mathrm{bA}$ & $4,49 \mathrm{bA}$ \\
\hline & 2006 & $17,39 \mathrm{aB}$ & $4,44 \mathrm{bA}$ & $3,89 \mathrm{bA}$ & $6,45 \mathrm{bA}$ & $4,28 \mathrm{bA}$ & $7,73 \mathrm{bA}$ & $5,54 \mathrm{bA}$ & $4,70 \mathrm{bA}$ \\
\hline \multirow{2}{*}{$\mathrm{K}_{\mathrm{SG}}\left(\mathrm{cm} \mathrm{s}^{-1}\right)$} & 2005 & $8,54 \mathrm{abB}$ & $11,06 \mathrm{aA}$ & $2,48 \mathrm{dA}$ & $3,89 \mathrm{cdA}$ & $3,57 \mathrm{dA}$ & $3,86 \mathrm{cdA}$ & $6,71 \mathrm{bcA}$ & $1,84 \mathrm{dA}$ \\
\hline & 2006 & $18,31 \mathrm{aA}$ & $6,01 \mathrm{bB}$ & $2,44 \mathrm{cdA}$ & $1,43 \mathrm{dA}$ & $2,73 \mathrm{cdA}$ & $1,79 \mathrm{cdA}$ & $3,13 \mathrm{cdB}$ & $3,71 \mathrm{cA}$ \\
\hline
\end{tabular}

(1)Médias seguidas de letras iguais, minúsculas na linha e maiúsculas na coluna, não diferem entre si pelo teste de t, a $5 \%$ de probabilidade. ${ }^{(2)} \mathrm{D}_{\mathrm{s}}$, densidade do solo; $\theta_{\mathrm{s}}$, umidade gravimétrica do solo na amostragem; PT, porosidade total do solo; Ma, macroporosidade; $\mathrm{Mi}_{\mathrm{e}}$, microporosidade efetiva; $\mathrm{S}_{\mathrm{e}}$, saturação efetiva; $\mathrm{K}_{\mathrm{SL}}$, condutividade hidráulica saturada de laboratório; $\mathrm{K}_{\mathrm{SG}}$, condutividade hidráulica saturada de campo. ${ }^{(3)} \mathrm{CE}$, cerrado nativo; $\mathrm{PC}$, pastagem contínua, com 19 anos de uso; P1, pastagem de Urochloa brizantha, com quatro anos de uso; P2, pastagem de U. brizantha, com dois anos de uso; P3, pastagem de $U$. brizantha, com três anos de uso; $\mathrm{C} 1$, soja sob plantio direto; $\mathrm{C} 2$, arroz em plantio convencional; $\mathrm{C} 3, U$. brizantha em consórcio com milho. 
as CRA dos outros sistemas se distinguiram significativamente da CRA do cerrado (Tabela 4). Diferentemente dos resultados encontrados por Marchão et al. (2007) e Borges et al. (2009), os resultados deste trabalho indicam que a CRA analisada individualmente não é um indicador sensível da qualidade física do solo.

Houve perda na qualidade física do solo em todos os sistemas, em comparação à pastagem contínua (Figura 2). As comparações realizadas pelo diagrama

Tabela 4. Valores de probabilidade do teste F, com três e dez graus de liberdade, nas comparações das curvas de umidade relativa entre os sistemas de uso e manejo, avaliados nas camadas de 0-20 cm (acima da diagonal) e 70-80 cm (abaixo da diagonal) de profundidade.

\begin{tabular}{lcccccccc}
\hline Sistemas $^{(1)}$ & \multicolumn{9}{c}{ Sistemas de uso e manejo } \\
\cline { 2 - 8 } & $\mathrm{CE}$ & $\mathrm{PC}$ & $\mathrm{P} 1$ & $\mathrm{P} 2$ & $\mathrm{P} 3$ & $\mathrm{C} 1$ & $\mathrm{C} 2$ & $\mathrm{C} 3$ \\
\hline $\mathrm{CE}$ & - & $<0,001$ & $<0,001$ & $<0,001$ & $<0,001$ & $<0,001$ & 0,001 & $<0,001$ \\
PC & 0,004 & - & $<0,001$ & 0,055 & 0,075 & 0,030 & $<0,001$ & $<0,001$ \\
P1 & $<0,001$ & 0,002 & - & $<0,001$ & $<0,001$ & $<0,001$ & $<0,001$ & 0,006 \\
P2 & 0,005 & 0,067 & 0,202 & - & 0,973 & 0,046 & $<0,001$ & 0,015 \\
P3 & 0,330 & $<0,001$ & 0,001 & $<0,001$ & - & 0,036 & $<0,001$ \\
C1 & $<0,001$ & $<0,001$ & 0,046 & 0,006 & 0,110 & - & $<0,001$ \\
C2 & 0,001 & 0,024 & 0,058 & 0,837 & $<0,001$ & $<0,001$ & - & $<0,011$ \\
C3 & 0,015 & 0,400 & 0,014 & 0,399 & $<0,001$ & $<0,001$ & 0,414 & $<0,001$ \\
\hline
\end{tabular}

${ }^{(1)} \mathrm{CE}$, cerrado nativo; PC, pastagem contínua, com 19 anos de uso; P1, pastagem de Urochloa brizantha, com quatro anos de uso; P2, pastagem de U. brizantha, com dois anos de uso; P3, pastagem de U. brizantha, com três anos de uso; C1, soja sob plantio direto; $\mathrm{C} 2$, arroz em plantio convencional; C3, U. brizantha em consórcio com milho.
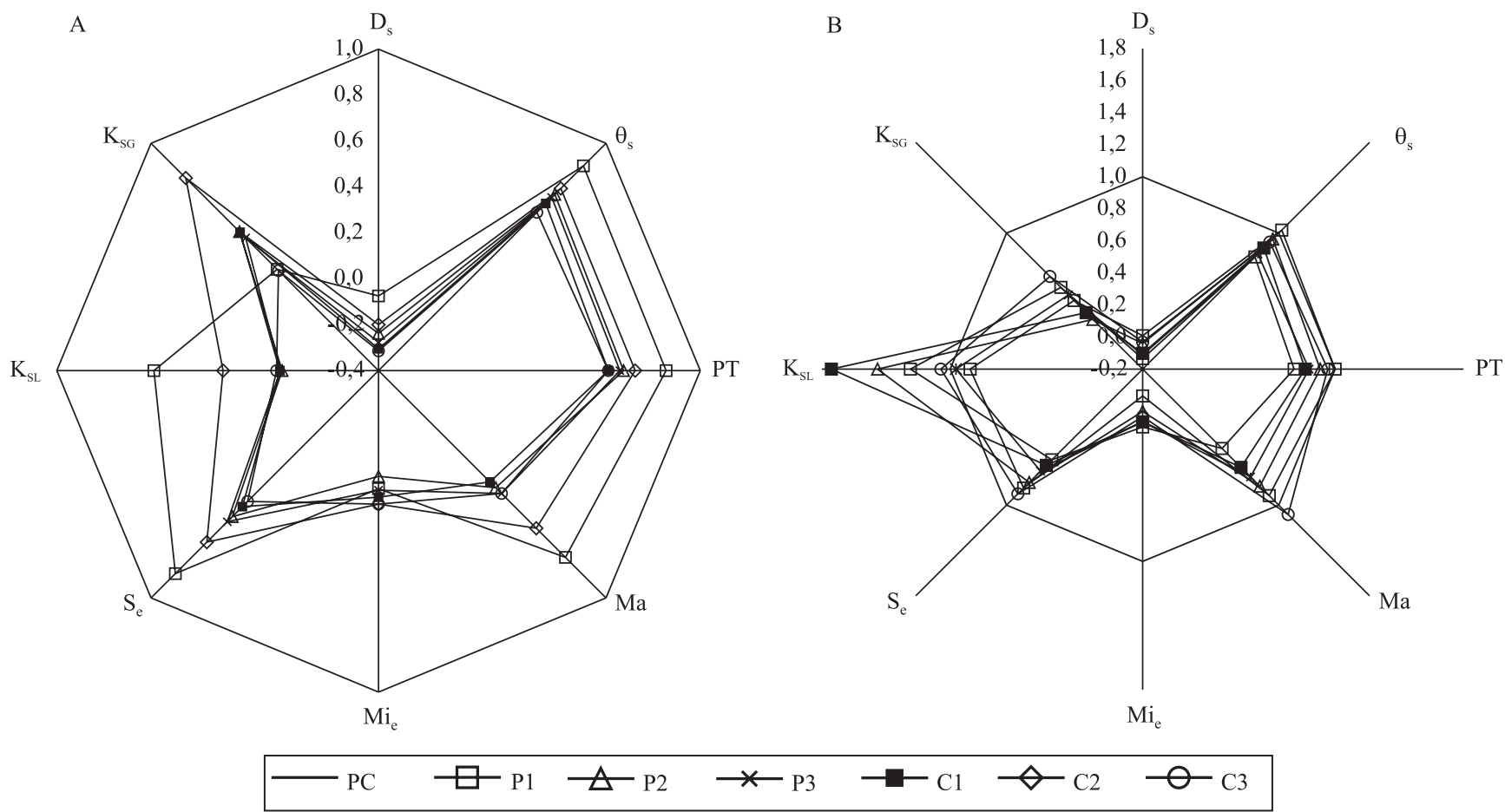

Figura 2. Diagrama comparativo da qualidade física do solo de áreas sob diferentes sistemas de manejo e uso, na profundidade 0-20 cm, para A, 2005 e $\mathrm{B}, 2006 . \mathrm{D}_{\mathrm{s}}$, densidade do solo; $\theta_{\mathrm{s}}$, umidade gravimétrica do solo na amostragem; PT, porosidade total do solo; $\mathrm{Ma}$, macroporosidade; $\mathrm{Mi}_{\mathrm{e}}$, microporosidade efetiva; $\mathrm{S}_{\mathrm{e}}$, saturação efetiva; $\mathrm{K}_{\mathrm{SL}}$, condutividade hidráulica saturada de laboratório; $\mathrm{K}_{\mathrm{SG}}$, condutividade hidráulica saturada de campo; PC, pastagem contínua, com 19 anos de uso; P1, pastagem de Urochloa brizantha, com quatro anos de uso; P2, pastagem de U. brizantha, com dois anos de uso; $\mathrm{P} 3$, pastagem de $U$. brizantha, com três anos de uso; $\mathrm{C} 1$, soja sob plantio direto; $\mathrm{C} 2$, arroz em plantio convencional; $\mathrm{C}$, $U$. brizantha em consórcio com milho. 
permitiram identificar mudanças nas propriedades físicas do Latossolo, quando ele foi submetido a diferentes sistemas de rotação entre lavoura de grãos e pastagens. Essas alterações foram verificadas, principalmente, nos parâmetros relacionados à porosidade do solo. No sistema P1, que permaneceu nos últimos quatro anos sob pastagem, não houve recuperação da estrutura do solo. Mesmo após quatro anos de cultivo sob pastagem, não foi possível recuperar a estrutura (macroagregação) perdida por consecutivos anos de preparo convencional antes da instalação do experimento.

$\mathrm{O}$ conceito de pastagens degradadas tem sido frequentemente utilizado para caracterizar as áreas sob pastagens de baixa produtividade no Cerrado. No entanto, deve-se ter cautela em relação ao uso deste conceito quanto à qualidade físico-hídrica. Apesar da degradação nas camadas superficiais dos solos ocupados pelas pastagens, entre os sistemas de uso da terra, a pastagem contínua foi a que apresentou a melhor qualidade física do solo.

\section{Conclusões}

1. Os sistemas de integração lavoura-pecuária avaliados provocam impactos negativos na qualidade física do solo, e a pastagem contínua, ainda que degradada, é a que resulta em melhor qualidade física do solo.

2. No sistema convencional de preparo do solo, ocorre incremento da densidade e redução da porosidade do solo.

3. A pastagem em rotação no sistema de integração lavoura-pecuária, mesmo após quatro anos, não favorece a qualidade física do solo em comparação à pastagem contínua.

\section{Agradecimentos}

Ao assistente de pesquisa da Embrapa Cerrados, Nelson Camargos Moreira, e aos estagiários Ângelo Valverde da Silva, Aracelly dos Santos Castro, João Paulo da Conceição Bispo, Leison Simão do Amaral, Luana Cristine da Silva Pinheiro e Paula Andréia Alvarenga, pelo apoio nas coletas de campo e análises; ao Institut de Recherche pour le Développement (França); à Universidade Federal de Goiás e ao Conselho Nacional de Desenvolvimento Científico e Tecnológico, pelo apoio financeiro e concessão de bolsa de doutorado.

\section{Referências}

ALBUQUERQUE, J.A.; SANGOI, L.; ENDER, M. Efeitos da integração lavoura-pecuária nas propriedades físicas do solo e características da cultura do milho. Revista Brasileira de Ciência do Solo, v.25, p.717-723, 2001.

BORGES, T.A.; OLIVEIRA, F.A.; SILVA, E.M. da; GOEDERT, W.J. Avaliação de parâmetros físico-hídricos de Latossolo Vermelho sob pastejo e sob cerrado. Revista Brasileira de Engenharia Agrícola e Ambiental, v.13, p.18-25, 2009.

BROCH, D.L. Integração agricultura-pecuária no Centro-Oeste do Brasil. In: ENCONTRO REGIONAL DE PLANTIO DIRETO NO CERRADO, 4., 1999, Uberlândia. Anais. Uberlândia: UFU/ APDC, 2000. p.53-60.

CLAESSEN, M.E.C. (Org.). Manual de métodos de análise de solo. 2.ed. rev. atual. Rio de Janeiro: Embrapa-CNPS, 1997. 212p. (Embrapa-CNPS. Documentos, 1).

FIDALSKI, J.; TORMENA, C.A.; CECATO, U.; BARBERO, L.M.; LUGÃO, S.M.B.; COSTA, M.A.T. Qualidade física do solo em pastagem adubada e sob pastejo contínuo. Pesquisa Agropecuária Brasileira, v.43, p.1583-1590, 2008.

GENUCHTEN, M.T. van. A closed-form equation for predicting the hydraulic conductivity of unsaturated soils. Soil Science Society of America Journal, v.44, p.892-898, 1980.

LANZANOVA, M.E.; NICOLOSO, R.S.; LOVATO, T.; ELTZ, F.L.F.; AMADO, T.J.C.; REINERT, D.J. Atributos físicos do solo em sistema de integração lavoura-pecuária sob plantio direto. Revista Brasileira de Ciência do Solo, v.31, p.1131-1140, 2007.

MACEDO, M.C.M. Integração lavoura e pecuária: o estado da arte e inovações tecnológicas. Revista Brasileira de Zootecnia, v.38, p.133-146, 2009.

MAGALHÃES, R.T. de; KLIEMANN, H.J.; OLIVEIRA, I.P. de. Evolução das propriedades físicas de solos submetidos ao manejo do sistema barreirão. Pesquisa Agropecuária Tropical, v.31, p.7-13, 2001.

MARCHÃO, R.L.; BALBINO, L.C.; SILVA, E.M. da; SANTOS JUNIOR, J. de D.G. dos; SÁ, M.A.C. de; VILELA, L.; BECQUER, T. Qualidade física de um Latossolo Vermelho sob sistemas de integração lavoura-pecuária no Cerrado. Pesquisa Agropecuária Brasileira, v.42, p.873-882, 2007.

MARCHÃO, R.L.; VILELA, L.; PALUDO, A.L.; GUIMARÃES JUNIOR, R. Impacto do pisoteio animal na compactação do solo sob integração lavoura-pecuária no Oeste Baiano. Planaltina: Embrapa Cerrados, 2009. 6p. (Embrapa Cerrados. Comunicado técnico, 163).

MESQUITA, M. da G.B. de F.; MORAES, S.O. A dependência entre a condutividade hidráulica saturada e atributos físicos do solo. Ciência Rural, v.34, p.963-969, 2004.

MUNIZ, L.C. Avaliação bio-econômica em sistema de integração lavoura-pecuária. 2007. 91p. Dissertação (Mestrado) - Universidade Federal de Goiás, Goiânia.

PRECHAC, F.G. Propriedades fisicas y erosion in rotaciones de cultivos y pasturas. Revista INIA Uruguay, n.1, p.127-140, 1992. 
REATTO, A.; BRUAND, A.; MARTINS, E. de S.; MULLER, F.; SILVA, E.M. da; CARVALHO JÚNIOR, O.A.; BROSSARD, M.; RICHARD, G. Development and origin of the microgranular structure in Latosols of the Brazilian Central Plateau: significance of texture, mineralogy, and biological activity. Catena, v.76, p.122-134, 2009.

REATTO, A.; SILVA, E.M. da; BRUAND, A.; MARTINS, E. de S.; LIMA, J.E.F.W. Validity of the centrifuge method for determining the water retention properties of tropical soils. Soil Science Society of America Journal, v.72, p.1547-1553, 2008.

REYNOLDS, W.D.; ELRICK, D.E. A laboratory and numerical assessment of the guelph permeameter method. Soil Science, v.144, p.282-299, 1987.

SALES, L.E. de O.; CARNEIRO, M.A.C.; SEVERIANO, E. da C.; OLIVEIRA, G.C. de; FERREIRA, M.M. Qualidade física de Neossolo Quartzarênico submetido a diferentes sistemas de uso agrícola. Ciência e Agrotecnologia, v.34, p.667-674, 2010.

SANO, E.E.; ROSA, R.; BRITO, J.L.S.; FERREIRA, L.G. Mapeamento semidetalhado do uso da terra do Bioma Cerrado. Pesquisa Agropecuária Brasileira, v.43, p.153-156, 2008.

SANTOS, G.G. Impacto de sistemas de integração lavoura-pecuária na qualidade física do solo. 2010. 122p. Tese (Doutorado) - Universidade Federal de Goiás, Goiânia.

SANTOS, G.G.; SILVEIRA, P.M. da; MARCHÃO, R.L.; BECQUER, T.; RIOS, A.J.W.; RODRIGUES, C. Descrição morfológica de perfis de solos da Fazenda Capivara da Embrapa Arroz e Feijão. Santo Antônio de Goiás: Embrapa Arroz e Feijão, 2010. 51p. (Embrapa Arroz e Feijão. Documentos, 258).

SILVA, C.L.; KATO, E. Efeito do selamento superficial na condutividade hidráulica saturada da superfície de um solo do Cerrado. Pesquisa Agropecuária Brasileira, v.32, p.213-220, 1997.

SILVA, E.M. da; AZEVEDO, J.A. de. Influência do período de centrifugação na curva de retenção de água em solos de Cerrado. Pesquisa Agropecuária Brasileira, v.37, p.1487-1494, 2002.

SILVA, E.M. da; AZEVEDO, J.A. de. Período de centrifugação adequado para levantamento da curva de retenção da água em solos do Bioma Cerrado. Planaltina: Embrapa Cerrados, 2001. 40p. (Embrapa Cerrados. Boletim de pesquisa e desenvolvimento, $8)$.

SPERA, S.T.; SANTOS, H.P. dos; FONTANELI, R.S.; TOMM, G.O. Integração lavoura e pecuária e os atributos físicos de solo manejado sob sistema plantio direto. Revista Brasileira de Ciência do Solo, v.33, p.129-136, 2009.

VILELA, L.; MARTHA JÚNIOR, G.B.; MARCHÃO, R.L.; GUIMARÃES JÚNIOR, R.; BARIONI, L.G.; BARCELOS, A. de O. Integração lavoura-pecuária. In: FALEIRO, F.G.; FARIAS NETO, A.L. de (Org.). Savanas: desafios e estratégias para o equilíbrio entre sociedade, agronegócio e recursos naturais. Planaltina: Embrapa Cerrados, 2008. v.1, p.933-962.

Recebido em 31 de julho de 2010 e aprovado em 27 de setembro de 2011 